\title{
Bayes Estimation of Parameter of Laplace Distribution Under a New LINEX-Based Loss Function
}

\author{
Lanping Li \\ School of Mathematics and Statistics, Hunan University of Finance and Economics, Changsha, China
}

\section{Email address:}

lilanping1981@163.com

To cite this article:

Lanping Li. Bayes Estimation of Parameter of Laplace Distribution Under a New LINEX-Based Loss Function. International Journal of Data Science and Analysis. Vol. 3, No. 6, 2017, pp. 85-89. doi: 10.11648/j.ijdsa.20170306.14

Received: October 27, 2017; Accepted: November 29, 2017; Published: December 26, 2017

\begin{abstract}
Loss function is one of the most topics in Bayesian analysis. The aim of this paper is to study the estimation of the shape parameter of Laplace distribution using Bayesian technique under a new loss function, which is a compound function of LINEX function. The Bayes estimator of the parameter is derived under the prior distribution of the parameter based on Gamma prior distribution. Furthermore, Monte Carlo statistical simulations illustrate that the Bayes estimators obtained under LINEX-based loss function is affected by the prior parameter and the value of the shape parameter of the LINEX-based loss function. But when the sample size is large, they have less influence on the estimation result.
\end{abstract}

Keywords: Laplace Distribution, Bayes Estimation, LINEX-Based Loss Function, Prior Distribution

\section{Introduction}

Laplace distribution is a commonly used distribution for modeling lifetime in engineering field. Because of its distribution with peak and thick tail, it is a more suitable distribution to describe the financial data than the normal distribution, so it has more research and application in the field of finance [1-7]. In recent years, the distribution has also been applied to image analysis, mechanical engineering and other fields [8-11].

The probability of Laplace distribution is as follows:

$$
f(x \mid \mu, \lambda)=\frac{1}{2 \lambda} \exp \left(-\frac{|x-\mu|}{\lambda}\right),-\infty<x<\infty
$$

where $\mu \in(-\infty,+\infty)$ is the location parameter and $\lambda>0$ is the scale parameter.

As an important class of statistical distributions, the statistical inference of parameters of Laplace distribution has attracted great attention. Iliopoulos and Balakrishnan [12] developed exact inference for the location and scale parameters of Laplace distribution based on their maximum likelihood estimators (MLEs) with a Type-II censored data. Gel [13] proposed a new test of fit for a Laplace distribution based on sample skewness and kurtosis and a robust L1estimator of scale about a sample median. Shi et al. [14] studied robust estimation for linear and mixture linear errorsin-variables regression models based on the relationship between the least absolute deviation criterion and MLE in a Laplace distribution. Rasheed and Al-Shareefi [15] studied the minimax estimation problem of Laplace scale parameters under the log error squared loss function. More studies can be found in [16-19].

Loss function plays important role in Bayesian statistical inference. Squared error loss function is the most often used function in Bayesian analysis. But in some reliability and lifetime field, overestimation and underestimation have different effects on decision results. In these situations, squared error loss function cannot work well. Then some scholars proposed several asymmetric loss functions [20-25], such as LINEX loss function, entropy loss function, etc. LINEX loss function is the most well-known asymmetric loss function. In recent years, a LINEX-based symmetric loss function is proposed by [27]. In literature [27], some excellent properties of the loss function are pointed out, and the Bayes estimation of the parameters of normal distribution and exponential distribution were studied under the loss function. In recent years, the reference [28-30] studied the Bayes estimation of Poisson distribution, Pareto distribution and Burr XII distribution parameter under the LINEX-based symmetric loss function.

This paper will also study Bayes estimation of the 
parameter $\theta=\lambda^{-1}$ under this LINEX-based symmetric loss function. This paper discussed the Bayes estimation with Quasi-information prior distribution of scale parameter of Laplace distribution estimation. Further, the admissibility of a class of estimators is also discussed.

\section{Preliminaries}

In the following discussion, we always suppose that $X_{1}, X_{2}, \cdots, X_{n}$ is a sample drawn from Laplace distribution (1), where the location parameter $\mu$ is known. Denote $x=\left(x_{1}, x_{2}, \cdots, x_{n}\right) \quad$ is the observation of sample $X=\left(X_{1}, X_{2}, \cdots, X_{n}\right)$ and $t=\sum_{i=1}^{n}\left|x_{i}-\mu\right|$ is the observation of statistics $T=\sum_{i=1}^{n}\left|X_{i}-\mu\right|$.

When given the observation $x=\left(x_{1}, x_{2}, \cdots, x_{n}\right)$, the likelihood function of parameter $\theta$ can be derived as follows:

$$
\begin{aligned}
l(x ; \theta) & =\prod_{i=1}^{n} f(x \mid \mu, \theta) \\
& =\prod_{i=1}^{n} \frac{\theta}{2} \exp \left(-\theta \cdot\left|x_{i}-\mu\right|\right) \\
& \propto \theta^{n} e^{-\theta t}
\end{aligned}
$$

By solving the log-likelihood function

$$
\frac{d \ln l(x ; \theta)}{d \theta}=n \ln \theta-\theta t=0,
$$

the MLE of $\theta$ is

$$
\hat{\delta}_{M L E}=\frac{n}{T}
$$

Let $T=\sum_{i=1}^{n}\left|X_{i}-\mu\right|$, then according to Rasheed and Al-Shareef [15], the statistic $T$ is distributed with Gamma distribution $\Gamma(n, \theta)$. Then

$$
E T=\frac{n}{\theta}
$$

Prior distribution and loss function play an important role in Bayesian statistical inference. In this paper, the prior distribution of the parameter is the Gamma prior distribution $\Gamma(\alpha, \beta)$, with probability density function

$$
\pi(\theta ; \alpha, \beta)=\frac{\beta^{\alpha}}{\Gamma(\alpha)} \theta^{\alpha-1} e^{-\beta \theta}, \quad \alpha, \beta>0, \theta>0
$$

As the most important component of Bayesian statistical inference, loss function plays a key role in choosing the appropriate loss function for robustness of statistical estimation. Because in the estimation of reliability and failure rate, often overestimate will bring greater losses, so although the squared error loss function is the most widely used loss functions, but the development of new symmetric and asymmetric loss function is also very necessary. The LINEX loss function is the most commonly used asymmetric loss function:

$$
L_{c}(\Delta)=e^{c \Delta}-c \Delta-1, \quad c \neq 0
$$

Where $\Delta=\delta-\theta$, and $\delta$ is an estimate of the parameter $\theta$. In the following discussion, we will discuss Bayesian estimation of scale parameter of Laplace under the LINEXbased loss function, which is also named compounded LINEX symmetric loss function and given as follows:

$$
L(\Delta)=L_{c}(\Delta)+L_{-c}(\Delta)=e^{c \Delta}+e^{-c \Delta}-2,
$$

Where $\Delta=\delta-\theta$ and $c>0$ is the shape parameter of $L(\Delta)$. Figure 1 gives the shape of the loss function with $c=1$.

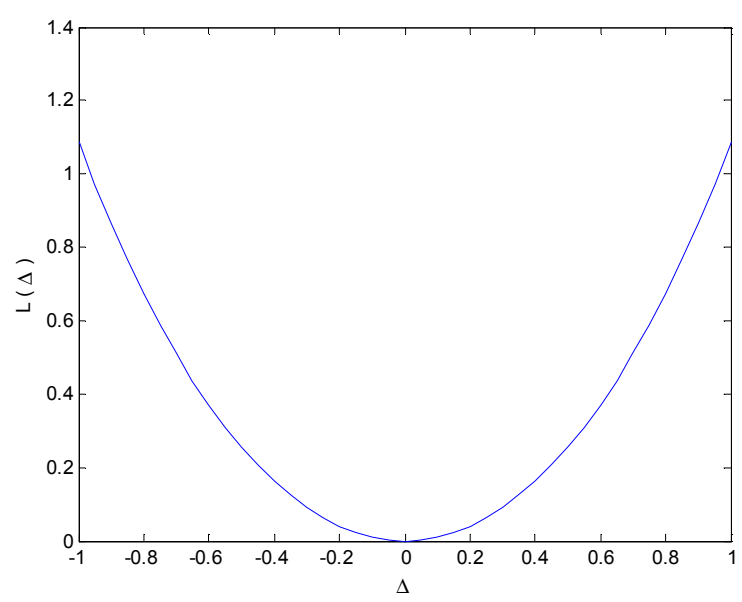

Figure 1. The shape of loss function $L(\Delta)$.

Lemma 1 Let $\delta$ is an estimator of the parameter $\theta$. For any prior distribution $\pi(\theta)$ of parameter $\theta$, under the LINEX-based loss function (6), the Bayes estimator of the parameter $\theta$ is

$$
\delta_{B}=\frac{1}{2 c} \ln \left(\frac{E\left(e^{c \theta} \mid X\right)}{E\left(e^{-c \theta} \mid X\right)}\right)
$$

And the Bayes estimator is unique, when assuming the Bayes risk $r(\delta)<+\infty$.

Proof. Under the LINEX-based loss function (7), the Bayes risk function of estimator $\delta$ is

$$
r(\delta)=E_{\theta}[E(L(\theta, \delta) \mid X)]
$$

To let $r(\delta)$ reach the minimum, we need $E(L(\theta, \delta) \mid X)$ to reach the minimum almost everywhere.

Because

$$
\begin{aligned}
E(L(\theta, \delta) \mid X) & =E\left(e^{c(\delta-\theta)}+e^{-c(\delta-\theta)}-2 \mid X\right) \\
& =e^{c \delta} E\left(e^{-c \theta} \mid X\right)+e^{-c \delta} E\left(e^{c \theta} \mid X\right)-2
\end{aligned}
$$


Therefore, one can only need the right side of the upper formula is minimized, then let $f(\delta)=e^{c \delta} E\left(e^{-c \theta} \mid X\right)+e^{-c \delta} E\left(e^{c \theta} \mid X\right)-2$.

It is easy to get

$$
\begin{gathered}
f^{\prime}(\delta)=c e^{c \delta} E\left(e^{-c \theta} \mid X\right)-c e^{-c \delta} E\left(e^{c \theta} \mid X\right), \\
f^{\prime \prime}(\delta)=c^{2} e^{c \delta} E\left(e^{-c \theta} \mid X\right)+c^{2} e^{-c \delta} E\left(e^{c \theta} \mid X\right) .
\end{gathered}
$$

Due to $f^{\prime \prime}(\delta)>0$, the Bayes estimator $\hat{\delta}_{B}$ of parameter $\theta$ satisfies $f^{\prime}(\delta)=0$. Then the Bayes estimator $\hat{\delta}_{B}$ of parameter $\theta$ is

$$
\delta_{B}=\frac{1}{2 c} \ln \left(\frac{E\left(e^{c \theta} \mid X\right)}{E\left(e^{-c \theta} \mid X\right)}\right)
$$

Now we prove the uniqueness of the evidence: To prove the uniqueness, as long as the proof of $r\left(\hat{\delta}_{B}\right)<+\infty$. According to known condition $r(\delta)<+\infty$ and the relation $r\left(\hat{\delta}_{B}\right)<r(\delta)$, the result $r\left(\hat{\delta}_{B}\right)<+\infty$ is obvious.

This completes the proof.

\section{Bayes Estimation}

This section will study the Bayes estimation of the known shape parameter of the Laplace distribution under the LINEX-based loss function.

Theorem 1. Suppose that $X_{1}, X_{2}, \cdots, X_{n}$ is a sample of size $\mathrm{n}$, which is drawn from Laplace distribution with probability density function (1). Here the location parameter $\mu$ is known. Denote $x=\left(x_{1}, x_{2}, \cdots, x_{n}\right)$ is the observation of sample $X=\left(X_{1}, X_{2}, \cdots, X_{n}\right)$ and $t=\sum_{i=1}^{n}\left|x_{i}-\mu\right|$ is the observation of statistics $T=\sum_{i=1}^{n}\left|X_{i}-\mu\right|$. The prior distribution of the parameter $\theta$ is the Gamma prior distribution (4). Then under the LINEX-based loss function, the Bayes estimator of the unknown shape parameter $\theta$ is

$$
\delta_{B}=\frac{n+\alpha}{2 c} \ln \left(\frac{\beta+T+c}{\beta+T-c}\right)
$$

Proof. Combining the likelihood function (2) with the Gamma prior probability density function (4), the posterior probability density function of $\theta$ can be derived using Bayes' Theorem as follows

$$
\begin{aligned}
h(\theta \mid x) & \propto l(\theta ; x) \cdot \pi(\theta) \\
& \propto \theta^{n} e^{-\theta t} \cdot \frac{\beta^{\alpha}}{\Gamma(\alpha)} \theta^{\alpha-1} e^{-\beta \theta} \\
& \propto \theta^{n+\alpha-1} e^{-(\beta+t) \theta}
\end{aligned}
$$

Then the posterior distribution of parameter $\theta$ is Gamma distribution with parameter $n+\alpha$ and $\beta+T$, noted by $\theta \mid X \sim \Gamma(n+\alpha, \beta+T)$.

That is the corresponding probability density function is

$$
h(\theta \mid x)=\frac{(\beta+t)^{n+\alpha}}{\Gamma(n+\alpha)} \theta^{(n+\alpha)-1} e^{-(\beta+t) \theta}
$$

Hence

$$
\begin{aligned}
& E[\exp (c \theta) \mid X] \\
& =\int_{0}^{\infty} \exp (c \theta) \frac{(\beta+T)^{n+\alpha}}{\Gamma(n+\alpha)} \theta^{(n+\alpha)-1} e^{-(\beta+T) \theta} d \theta \\
& =\int_{0}^{\infty} \frac{(\beta+T)^{n+\alpha}}{\Gamma(n+\alpha)} \theta^{(n+\alpha)-1} e^{-(\beta+T-c) \theta} d \theta \\
& =\frac{(\beta+T)^{n+\alpha}}{\Gamma(n+\alpha)} \cdot \frac{\Gamma(n+\alpha)}{(\beta+T-c)^{n+\alpha}} \\
& =\left(\frac{\beta+T}{\beta+T-c}\right)^{n+\alpha} \\
& E[\exp (-c \theta) \mid X] \\
& =\int_{0}^{\infty} \exp (-c \theta) \frac{(\beta+T)^{n+\alpha}}{\Gamma(n+\alpha)} \theta^{(n+\alpha)-1} e^{-(\beta+T) \theta} d \theta \\
& =\int_{0}^{\infty} \frac{(\beta+T)^{n+\alpha}}{\Gamma(n+\alpha)} \theta^{(n+\alpha)-1} e^{-(\beta+T+c) \theta} d \theta \\
& =\frac{(\beta+T)^{n+\alpha}}{\Gamma(n+\alpha)} \cdot \frac{\Gamma(n+\alpha)}{(\beta+T+c)^{n+\alpha}} \\
& =\left(\frac{\beta+T}{\beta+T+c}\right)^{n+\alpha}
\end{aligned}
$$

Then according to Lemma1, under the LINEX-based loss function (7), the Bayes estimator of $\theta$ can be derived as follows

$$
\begin{aligned}
\delta_{B} & =\frac{1}{2 c} \ln \left(\frac{E\left(e^{c \theta} \mid X\right)}{E\left(e^{-c \theta} \mid X\right)}\right) \\
& =\frac{1}{2 c} \ln \left[\left(\frac{\beta+T}{\beta+T-c}\right)^{n+\alpha} /\left(\frac{\beta+T}{\beta+T+c}\right)^{n+\alpha}\right] \\
& =\frac{n+\alpha}{2 c} \ln \left(\frac{\beta+T+c}{\beta+T-c}\right) .
\end{aligned}
$$

\section{Numerical Simulation}

Using Monte Carlo statistical simulation, this paper generate random sample from the Laplace distribution (1) with parameters of $\mu=1, \theta=1.0$ and sample sizes $n=10,20$, $30,50,75,100$. Repeat the simulation experiment $\mathrm{N}=5000$ times and use the estimate of the mean $\hat{\theta}=\frac{1}{N} \sum_{i=1}^{N} \hat{\theta}_{i}$ as the estimated values of the parameter $\theta$, and use the estimated 
mean square error $E R(\hat{\theta})=\frac{1}{N} \sum_{i=1}^{N}\left(\hat{\theta}_{i}-\theta\right)^{2}$ as a measure of good estimation standards. Here $\hat{\theta}_{i}$ is the ith estimate of the parameter $\theta$. The estimated and mean square errors of the maximum likelihood and Bayes estimators of the parameters are shown in Tables 1 and 2, in which the mean square error is in parentheses.

Table 1. Estimation and mean square error under different sample sizes ( $\alpha=1$ and $\beta=1$ ).

\begin{tabular}{llllll}
\hline $\mathbf{n}$ & $\mathbf{1 0}$ & $\mathbf{2 0}$ & $\mathbf{5 0}$ & $\mathbf{7 5}$ & $\mathbf{1 0 0}$ \\
\hline$\delta_{M L}$ & $1.1128(0.1611)$ & $1.0587(0.0697)$ & $1.0210(0.0214)$ & $1.0131(0.0143)$ & $1.0097(0.0104)$ \\
$\delta_{B}(\mathrm{c}=0.5)$ & $1.0913(0.1170)$ & $1.0531(0.0607)$ & $1.0202(0.0204)$ & $1.0128(0.0139)$ & $1.0095(0.0102)$ \\
$\delta_{B}(\mathrm{c}=1.0)$ & $1.0948(0.1202)$ & $1.0538(0.0611)$ & $1.0203(0.0204)$ & $1.0128(0.0139)$ \\
$\delta_{B}(\mathrm{c}=1.5)$ & $1.1009(0.1258)$ & $1.0551(0.0617)$ & $1.0205(0.0205)$ & $1.0129(0.0139)$ & $1.0095(0.0102)$ \\
\hline
\end{tabular}

Table 2. Estimation and mean square error under different sample sizes ( $\alpha=0.5$ and $\beta=1.5$ ).

\begin{tabular}{llllll}
\hline $\mathbf{n}$ & $\mathbf{1 0}$ & $\mathbf{2 0}$ & $\mathbf{5 0}$ & $\mathbf{7 5}$ & $\mathbf{1 0 0}$ \\
\hline$\delta_{M L}$ & $1.1134(0.1729)$ & $1.0499(0.0630)$ & $1.0226(0.0226)$ & $1.0133(0.0141)$ & $1.0093(0.0098)$ \\
$\delta_{B}(\mathrm{c}=0.5)$ & $0.9880(0.0837)$ & $0.9942(0.0457)$ & $1.0015(0.0199)$ & $0.9996(0.0130)$ & $0.9991(0.0092)$ \\
$\delta_{B}(\mathrm{c}=1.0)$ & $0.9908(0.0853)$ & $0.9949(0.0459)$ & $1.0016(0.0199)$ & $0.9996(0.0130)$ & $0.9991(0.0092)$ \\
$\delta_{B}(\mathrm{c}=1.5)$ & $0.9957(0.0882)$ & $0.9960(0.0462)$ & $1.0018(0.0199)$ & $0.9997(0.0130)$ & $0.9992(0.0092)$ \\
\hline
\end{tabular}

From Table 1 and Table 2 it can be seen that the Bayes estimators obtained under LINEX-based loss function is affected by the value of the shape parameter estimation of $\mathrm{c}$. When sample size $\mathrm{n}$ is small, the value of parameter has a larger influence on the estimation results, but with the increase of sample size $n$, the value of mean square error estimate is decreasing. When the sample size $n$ is larger than 50 , the influence of is very small, and sometimes can be ignore and the estimated value of more and more close to the true values of parameters. At the same time, compared with Table 1 and Table 2, we find that when the sample size $\mathrm{n}$ is large, the change of prior distribution has less influence on the estimation result.

\section{Conclusion}

Because of the loss function plays an important role in the Bayes statistical inference, construction of new symmetric loss function plays an important role in the enrichment and development of Bayes statistical inference theory, this paper based on the LINEX loss function, a new class of LINEXbased symmetric loss function is proposed. In this paper, the Bayes estimation of the parameter is derived under the loss function. Furthermore, the Bayes estimation of scale parameter of Laplace distribution is further studied. The Bayes estimators of the parameters are derived under the prior distribution of the parameters as the Gamma prior distribution. Finally, the properties of the estimators are investigated by means of Monte Carlo statistical simulation.

\section{Acknowledgements}

The author is grateful to the reviewers for a very careful reading of the manuscript and the suggestions that lead to the improvement of the paper. This study is partially supported by 933rd items of Education Reform Project in Hunan
Province in 2016.

\section{References}

[1] Linden M. A model for stock return distribution [J]. International Journal of Finance \& Economics, 2001, 6 (2): 159-169.

[2] Komunjer I. Asymmetric power distribution: Theory and applications to risk measurement [J]. Journal of Applied Econometrics, 2007, 22 (5): 891-921.

[3] Chi Y. Analysis of the expected discounted penalty function for a general jump-diffusion risk model and applications in finance $[\mathrm{J}]$. Insurance Mathematics \& Economics, 2010, 46 (2): $385-396$.

[4] Chen Q, Gerlach R, Lu Z. Bayesian Value-at-Risk and expected shortfall forecasting via the asymmetric Laplace distribution [J]. Computational Statistics \& Data Analysis, 2012, 56 (11): 3498-3516.

[5] Zhang H. Study on financial market risk measurement based on asymmetric Laplace distribution [J]. American Journal of Theoretical and Applied Statistics, 2015, 4 (4): 264.

[6] Kaldasch J. Evolutionary model of stock markets [J]. Physica A Statistical Mechanics \& Its Applications, 2015, 415: 449462.

[7] Autchariyapanitkul K., Chanaim S., Sriboonchitta S. Quantile regression under asymmetric Laplace distribution in capital asset pricing model [J]. Studies in Computational Intelligence, 2015, 583: 219-231.

[8] Cui X, Yu K, Lu S. Direction finding for transient acoustic source based on biased TDOA measurement [J]. IEEE Transactions on Instrumentation \& Measurement, 2016, 65 (11): 2442-2453.

[9] Zhang L, Wang H, Qiao Z J. Resolution enhancement for ISAR imaging via improved statistical compressive sensing [J]. Eurasip Journal on Advances in Signal Processing, 2016, 2016 (1): 80. 
[10] Fang R, Al-Bayaty R, Wu D. BNB Method for No-Reference Image Quality Assessment [J]. IEEE Transactions on Circuits \& Systems for Video Technology, 2017, 27 (7): 1381-1391.

[11] Cui J, Wang S, Wang S, et al. Hybrid Laplace distributionbased low complexity rate-distortion optimized quantization [J]. IEEE Transactions on Image Processing, 2017, 26 (8): 3802-3816.

[12] Iliopoulos G, Balakrishnan N. Exact likelihood inference for Laplace distribution based on Type-II censored samples [J]. Journal of Statistical Planning \& Inference, 2011, 141 (3): 1224-1239.

[13] Gel Y R. Test of fit for a Laplace distribution against heavier tailed alternatives [J]. Computational Statistics \& Data Analysis, 2010, 54 (4): 958-965.

[14] Shi J, Chen K, Song W. Robust errors-in-variables linear regression via Laplace distribution [J]. Statistics \& Probability Letters, 2014, 84 (1): 113-120.

[15] Rasheed H A, Al-Shareefi E F. Minimax estimation of the scale parameter of Laplace distribution under squared-log error loss function [J]. Mathematical Theory \& Modeling, 2015, 5 (1): 183-193.

[16] Jiang C F, Peng H Y, Yang Y K. Tail variance of portfolio under generalized Laplace distribution [J]. Applied Mathematics \& Computation, 2016, 282 (C): 187-203.

[17] Alomari A I, Haq A. Entropy estimation and goodness-of-fit tests for the inverse Gaussian and Laplace distributions using paired ranked set sampling [J]. Journal of Statistical Computation \& Simulation, 2015, 86 (11): 1-11.

[18] Rasheed H A. Bayesian and non-Bayesian estimation for the scale parameter of Laplace distribution [J]. Advances in Environmental Biology, 2016, 9 (14): 226-232.

[19] Liu H B, Yin T, Wang C. E-Bayes estimation of Laplace distribution parameter under Q-symmetric entropy loss function [J]. Applied Mechanics \& Materials, 2014, 596: 301304.

[20] Sadek A, Sultan K S, Balakrishnan N. Bayesian estimation based on ranked set sampling using asymmetric loss function [J]. Bulletin of the Malaysian Mathematical Sciences Society, 2015, 38 (2): 707-718.
[21] Han W, Rajan P, Frazier P I, et al. Bayesian group testing under sum observations: A parallelizable 2-approximation for entropy loss [J]. IEEE Transactions on Information Theory, 2017, 63 (2): 915-933.

[22] Nayak T K, Sinha B. An appreciation of balanced loss functions via regret loss [J]. Communications in Statistics Theory and Methods, 2015, 44 (3): 607-616.

[23] Nematollahi N. Admissible and minimax estimation of the parameter of the selected Pareto population under squared log error loss function [J]. Statistical Papers, 2017, 58 (2): 319339 .

[24] Li L. A Bayesian algorithm of wireless sensor network link selection under asymmetric loss function[J]. Research Journal of Applied Sciences Engineering \& Technology, 2016, 12(2):249-252.

[25] Li L. Bayes estimation of Topp-Leone distribution under symmetric entropy loss function based on lower record values [J]. Science Journal of Applied Mathematics and Statistics, 2016, 4 (6): 284-288.

[26] Li J, Ren H. Estimation of one parameter exponential family under a precautionary loss function based on record values [J]. International Journal of Engineering and Manu- facturing, 2012, 2 (3): 75-81.

[27] Zhang R. The Parameter Estimation under the Compound LINEX Symmetric Loss Function [D]. Dalian: Dalian University of Technology, 2010. (In Chinese).

[28] Wei C D, Wei S, Su H. Bayes estimation and application of Poisson distribution parameter under compound LINEX symmetric loss [J], Statistics and Decision, 2010 (7): 156-157. (In Chinese).

[29] Wei C D, Wei S, Su H. E-Bayes estimation of shape parameter of Pareto distribution under compound LINEX symmetric loss and its application [J]. Statistics and Decision, 2009 (17): 7-9. (In Chinese).

[30] Wei S., Li Z. N. Bayes estimation of Burr XII distribution parameter in the composite LINEX loss of symmetry [J]. Applied Mathematics A Journal of Chinese Universities, 2017, 32 (1): 49-54. (In Chinese). 